livraisons

d'Histoire

de l'Architecture

\section{Livraisons de l'histoire de l'architecture}

$22 \mid 2011$

Émotions patrimoniales II

\title{
Des « Pics émotionnels » : l'exemple de Francfort
}

Summits of Emotion, the Example of Frankfurt

« Emotionale Spitzenwerte » : das Frankfurter Beispiel

\section{Markus Dauss}

\section{OpenEdition}

\section{Journals}

Édition électronique

URL : http://journals.openedition.org/lha/288

DOI : 10.4000/lha.288

ISSN : 1960-5994

Éditeur

Association Livraisons d'histoire de l'architecture - LHA

Édition imprimée

Date de publication : 10 décembre 2011

Pagination : $33-50$

ISSN : 1627-4970

Référence électronique

Markus Dauss, « Des «Pics émotionnels » : l'exemple de Francfort », Livraisons de l'histoire de l'architecture [En ligne], 22 | 2011, mis en ligne le 10 décembre 2013, consulté le 19 avril 2019. URL: http://journals.openedition.org/lha/288 ; DOI : 10.4000//ha.288

Ce document a été généré automatiquement le 19 avril 2019

Tous droits réservés à l'Association LHA 


\title{
Des « Pics émotionnels » : l'exemple de Francfort
}

\author{
Summits of Emotion, the Example of Frankfurt \\ « Emotionale Spitzenwerte» : das Frankfurter Beispiel
}

Markus Dauss

1 Le rapport entre patrimoine et émotion peut être illustré en se fondant sur l'architecture des buildings. En effet, un lien évident peut être établi entre émotion et hauteur des constructions. En tant que représentation emblématique de la construction moderne, la notion de hauteur se trouve plus aisément chargée de points culminants émotionnels plutôt que de valeurs sublimées de l'ancienneté telles que les monuments les recèlent. Or, depuis peu, l'architecture des buildings et les discours concernant la protection des monuments semblent se rapprocher de plus en plus. Cela se manifeste surtout en examinant les bâtiments de grande hauteur, indépendamment de leurs exploitations respectives, et s'apparente au concept classique de monument ${ }^{1}$. C'est pourquoi il sera indispensable ici d'expliciter de manière exemplaire le rapport entre les géants architectoniques et le discours sur les monuments historiques, sans oublier le rôle et la fonction des émotions.

2 J'aborderai d'abord le rapport entre la construction en hauteur et l'évocation d'émotions. En Allemagne la dimension émotionnelle des buildings peut être suivie de façon paradigmatique et plus lisible qu'aux États-Unis dont l'architecture en hauteur est souvent dite «naturelle ». Déjà la réception presque euphorique de l'architecture d'outreAtlantique des buildings dans les années 20 en Allemagne - à cette époque encore largement conçue comme un devoir public - provoquait un scepticisme important ${ }^{2}$.Par conséquent le débat restait vivant et clairement explicité. Et même si après la deuxième guerre mondiale l'influence américaine germait bien sur les terres allemandes - surtout dans certaines villes - les buildings ont toujours été l'épicentre de luttes fortement émotionnelles pour le monopole d'interprétation que détient le discours architectural et urbain, et cela reste toujours d'actualité. Les buildings considérés en tant que véritables monuments, concept qui engloberait alors leur protection, demeurent très rares. 
3 À Francfort on comprend à quel point tant la construction que la disparition des buildings ont suscité d'émotions. Cela vaut d'autant plus que les buildings y acquièrent actuellement un statut objectif, c'est-à-dire institutionnalisé, de monument historique ${ }^{3}$. Or Francfort est la métropole la moins importante d'Allemagne en superficie et en population ; elle se définit d'après sa croissance en hauteur, et elle est très soucieuse de sa réputation de métropole européenne des gratte-ciels >. Le fait que son record de hauteur ait déjà été battu par Moscou (Federation Tower) ou qu'elle doive - probablement - bientôt céder à Londres son statut unique de site européen de building est conçu par des commentateurs anxieux comme une véritable menace pour son image ${ }^{4}$. Cependant en tant que métropole affirmée, Francfort n'est pas forcément tenue d'avoir des « superlatifs en hauteur ", mais elle peut désormais - de plus en plus - prendre en compte la qualité architectonique de ses constructions afin de conserver sa beauté et sa cohérence et maintenir ainsi dans l'ensemble de la silhouette urbaine ${ }^{5}$ une infrastructure compacte, urbaine et culturelle, assurant à ses habitants une bonne qualité de vie ${ }^{6}$. L'utilisation monoculturelle des buildings par le service tertiaire est de nos jours, paradoxalement, considérée comme un aspect négatif $\mathrm{du}$ site et ce même par des entreprises internationales ${ }^{7}$. D'un autre point de vue, l'histoire de l'art scientifique a reconnu ce type de silhouette urbaine et lui a attribué le titre d'œuvre d'art de qualité à part entière ${ }^{8}$. Malgré de telles orientations vers le qualitatif dans les discours autour des buildings à Francfort, chaque projet pour une nouvelle tour touche les esprits et les âmes. La résistance contre les buildings a toujours été la plus forte à Francfort; la ville mène des débats empreints d'affectivité sur sa physionomie en perpétuel changement. Cela vaut toujours même si les acteurs de l'opposition, les arguments et les formes du débat ont changé : les débatteurs se sont rendus compte que les positionnements traditionnels entre (Mainhattan〉 (terme euphorique) et (Bankfurt) ou «Krankfurt» (termes péjoratifs) s'étaient modifiés. Enfin, plus qu'un demi-siècle d'architecture de buildings propose quand même une grande diversité de matériaux et une distance historique pour adopter un discours plus nuancé.

4 Le cas que je présenterai par la suite est exemplaire pour exprimer la convergence des discours sur le patrimoine et sur la dimension émotionnelle. Il comprend plusieurs phases historiques différentes et a de ce fait exerce une influence sur la complexité du débat. Des lignes de front assez mobiles et maints retournements affectifs en sont le résultat. En outre notre exemple fait partie d'une discussion actuelle très animée sur la physionomie de Francfort en général. Au fond cela a toujours à voir avec les bouleversements traumatiques des villes allemandes depuis la deuxième guerre mondiale, villes qui ont toutes été marquées par une restructuration fondamentale ${ }^{9}$ contrairement à la plupart des villes en France. Il est essentiel que l'architecture de la reconstruction du Francfort d'après-guerre - qui a duré jusqu'aux années 70 - puisse maintenant faire valoir une valeur d'ancienneté, ce que les nouveaux projets de construction reflètent d'ores et déjà. Les démolitions sont à l'ordre du jour, surtout celles de la première génération des buildings qui s'est terminée vers la fin des années $50^{10}$. La révolution des tours «mange » alors ses enfants au moment même où ils sont assez vieux pour être considérés comme monuments.

5 Cette tension ne semble pas que la conséquence d'un vieillissement naturel de ces immenses tours, si modernes à l'époque ${ }^{11}$. Il y a de nouveaux aspects dans ce processus : l'innovation urbanistique initiale qui présidait à la construction des buildings après la guerre doit de nos jours d'une part maintenir sa place contre des projets de 
modernisation qui semblent plus justifiés économiquement ou stratégiquement (par rapport à l'image de la ville). D'autre part, le fonctionnalisme de la première génération des buildings entre aussi en conflit avec des projets actuels étiquetés comme "guérison " de la ville, concepts souvent imprégnés d'une certaine nostalgie. Contrairement à ces " mesures de guérison », on étiquette maintenant les "vieux » buildings de la première génération de l'après-guerre souvent comme «décidément non historiques ». Par conséquent, les exigences souvent brutales d'une économie globalisée ne s'accordent pas à une culture locale opposée. Car les représentants de l'économie ont souvent appris à intégrer symboles et idéaux d'une identité locale dans leurs projets, intégration considérée comme « indispensable » et typique de la " glocalisation ». En revanche autant la critique que la résistance contre ce nouvel amalgame maintiennent l'idée d'une modernité internationale ou - tout au moins - de sa continuation fonctionnaliste et revendiquent sa valeur proprement historique ${ }^{12}$.

Cette nouvelle situation s'applique surtout à la démolition du building de la mutualité Zürich (Zürich-Haus) en 2001, remplacée par la tour de l'Opéra (Opernturm) en construction depuis 2007, qui sera examinée dans ce qui suit sous l'angle de sa dimension émotionnelle. Le Zürich-Haus, construit en 1958 qui se trouvait à l'ouest de la place de l'Opéra au delà du Reuterweg, fut classé monument historique en 1988. En vertu des demandes et des instigations juridiques du côté du consortium propriétaire, et malgré les protestations du côté de la commission régionale du patrimoine et des sites, cette protection fut annulée par le ministère de la culture de Hesse pour une période de cinq ans en 1991 ainsi qu'en $1996^{13}$. Le rapport entre cet exemple et la question fondamentale posée dans cet article parait évident: le type du building particulièrement chargé d'émotions fut d'abord revalorisé comme monument culturel, puis finalement détruit malgré son récent statut d'objet patrimonial. (ill.1)

7 Le fait de démolir un bâtiment considéré comme monument culturel a contribué à son statut déjà perçu comme "icône de la reconstruction » et l'a transformé en martyr architectural. En revanche, et cela explique aussi la communication des préconisateurs, justifiant la procédure radicale et chargeant ce nouveau projet d'une émotivité positive : dans cette perspective les préconisateurs de cette démolition, loin de vouloir absolument gommer un des symboles les plus établis du paysage urbain de Francfort d'après guerre, avaient « simplement » l'intention de valoriser une identité locale. Or non seulement les partisans mais aussi les opposants de cette démolition trouvèrent des canaux médiatiques auprès à la fois des grands journaux régionaux et des publications suprarégionales de Francfort : le FAZ (Frankfurter Allgemeine Zeitung), quotidien plutôt conservateur basé sur le libéralisme économique qui accorda à l'architecte du nouveau bâtiment (Opernturm), Christoph Mäckler, un espace d'expression généreux ainsi que le FR (Frankfurter Rundschau), portail destiné à un public urbain plutôt opposant et gauchiste ${ }^{14}$. Dans la couverture médiatique et dans les commentaires de ces deux journaux antagonistes s'est élaborée une convergence exemplaire entre les moyens de communication "légers » et l'architecture comme moyen de communication «lourd», tous deux engendrant une présence publique, suscitant et traitant alors des émotions ${ }^{15}$. 


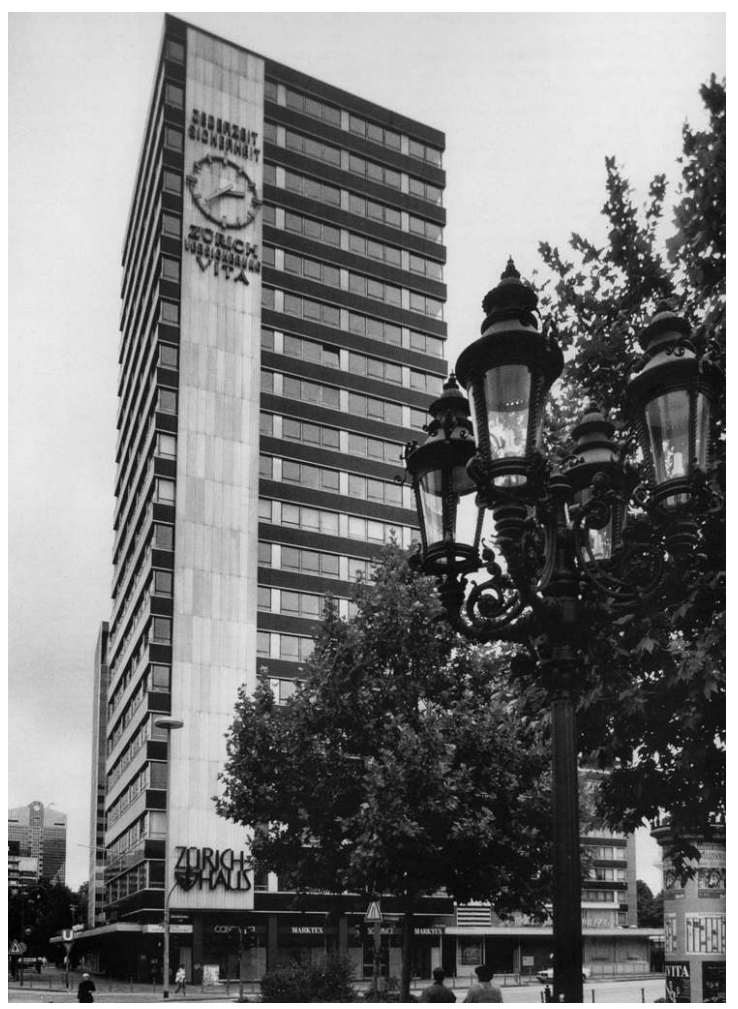

SOURCE : DETLEF JANIK (ÉD.), HOCHHÄUSER IN FRANKFURT. WETTLAUF ZU DEN WOLKEN, FRANKFURT AM MAIN, SOCIETÄTS-VERLAg, 1995

8 Pour la compréhension de la dimension affective de ce débat il sera essentiel de fournir quelques informations complémentaires. Ces faits devront montrer clairement à quel point l'objet en question du débat comporte un aspect identitaire également à travers son histoire. Revalorisée par la présence américaine après la seconde guerre mondiale, la ville de Francfort est rapidement devenue la métropole commerciale et économique de la zone ouest de l'Allemagne ${ }^{16}$. Elle n'était pas devenue une capitale comme la plupart l'avait espéré jusqu'en 1949, mais elle fut tout de même le quartier général puis le centre européen des troupes américaines.

Dans les années 50 , après une réorganisation municipale axée sur un développement plus économique que politique, des jalons pour la promotion de l'économie financière furent posés pour le site de Francfort ${ }^{17}$. La construction de plusieurs buildings pendant cette période à Francfort, fortement détruit par la guerre, ne paraît donc pas étonnante. Celleci était même souhaitée dans l'optique d'une urbanisation planifiée par la ville ${ }^{18}$. Aussi le lourd héritage néoclassique d'une architecture, trace au sol représentative des Nazis ${ }^{19}$, passait pour largement discrédité et inadmissible en raison de réflexes de refoulements collectifs. Donc les buildings de la première génération et leur apparence particulièrement humble étaient très bien reçus, non seulement en tant que preuves d'une mentalité pro américain ${ }^{20}$, mais aussi comme marques d'une internationalisation et d'une réparation symbolique en faveur des exilés ${ }^{21}$. Les raisons pour cela étaient (anti)émotionnelles: les buildings déployaient non seulement l'attitude objective et neutre souhaitée à un niveau mental, mais évoquaient aussi un geste calme opportun du point de vue de la psychologie collective. 
10 La préservation proportionnelle de pierres en façades (plutôt que des façades généreusement vitrées) put encore être considérée comme une référence au canon classique des proportions. Même la division des buildings des années 50 présentant un socle - allégé par des arcades et des piliers -, une partie principale de construction et enfin les parties hautes en forme d'un acrotère moderne voire d'un toit en surplomb ${ }^{22}$, rappelait les modèles canoniques d'architecture ou même l'ordre classique. En Allemagne la construction des buildings dans les années 20 avait déjà été déterminée par de telles caractéristiques $^{23}$. Par ce mélange qui combinait des éléments établis à une altitude inhabituelle et une trame rationnelle, donc des paramètres modernes, les édifices d'après-guerre auraient pu se présenter comme des incarnations parfaites de la "modernisation conservatrice», valeur typique de l'époque. On essayait alors d'éviter des effets de choc concernant l'altitude et la masse ${ }^{24}$.

11 La décision de la politique municipale de s'engager dans une construction de «buildings modérés » impliqua des auto-obligations envers le «bien commun », surtout au niveau de la physionomie urbaine, de l'économie du terrain et des problématiques du trafic. Les réactions émotionnelles du public face aux édifices ne jouent pas un rôle essentiel dans ces réflexions planificatrices. En effet d'après les documents concernant les réactions du public, les apparitions isolées, comme le langage des formes, entraînaient que les édifices étaient perçus comme des gestes non excessifs d'une modernisation consensuelle et prudente, considérée comme inévitable ${ }^{25}$. Au lieu d'un discours hautement affectif dominait une accoutumance à leur présence qui était la promesse d'un meilleur avenir sans que cela ne propage une césure fondamentale ${ }^{26}$. Pour représenter les symboles du miracle économique comme suite quasi-naturelle de traditions d'avant-guerre en escamotant l'époque nazie, les maîtres d'ouvrage - institutions municipales et publiques ou entreprises - et les responsables politiques suggérèrent pendant leurs discours d'inauguration ce point de vue par des constructions historiques relativement «acrobatiques ». À défaut de points de départ concrets, on évoquait des continuités institutionnelles ou géographiques, souvent de manière très artificielle ${ }^{27}$. (ill.2) 


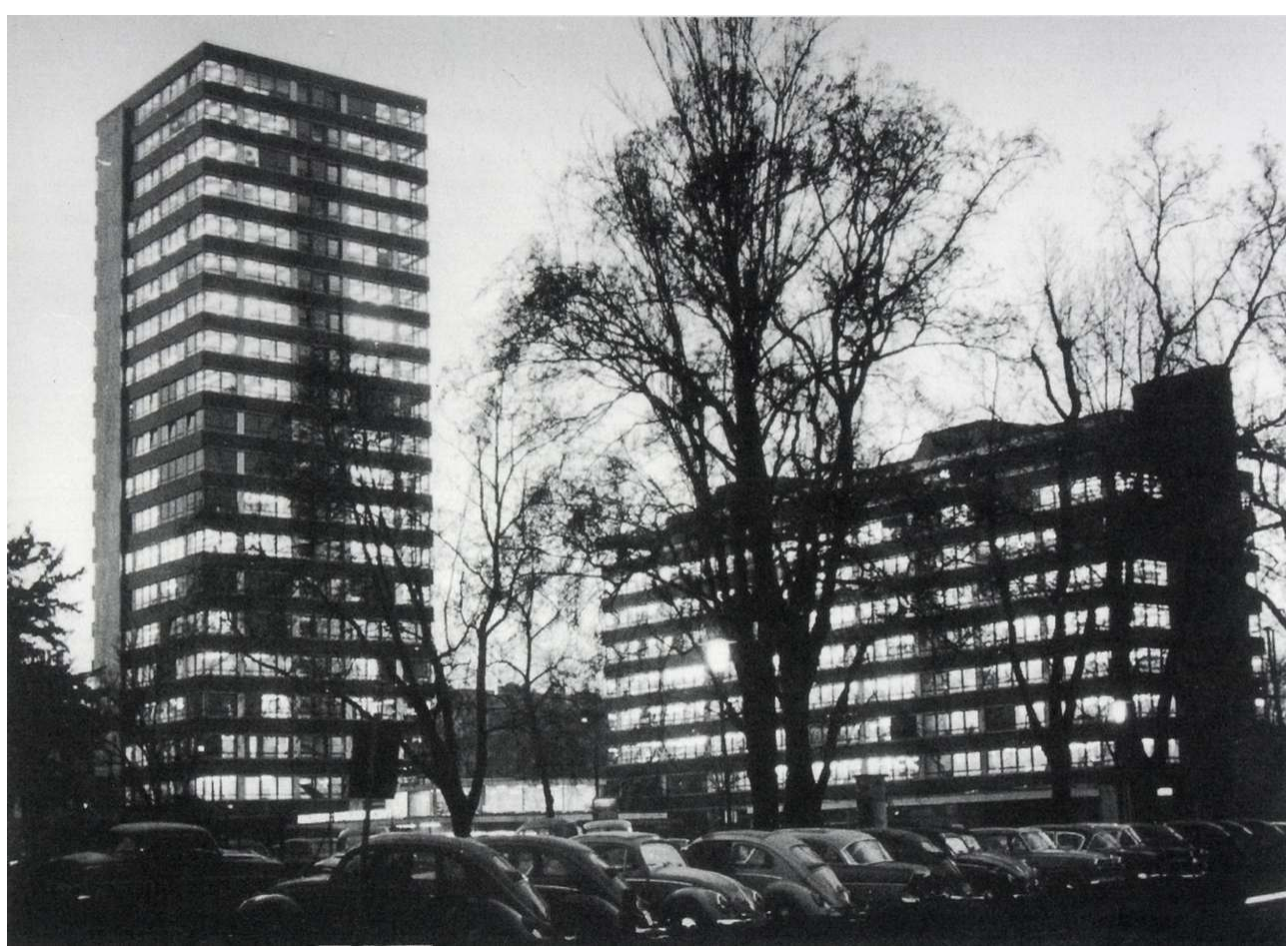

SOURCE : DIETER BARTETZKO (ÉD.), SPRUNG IN DIE MODERNE. FRANKFURT AM MAIN. DIE STADT DER 50ER JAHRE, FRANKFURT AM MAIN/NEW YORK, CAMPUS 1994

Le building que le groupe suisse Zürich-Versicherung avait commandé en 1958 aux architectes Werner Stücheli (Suisse) et Udo von Schauroth (Frankfurt) échappa initialement à ce consensus de «normalisation 〉 des buildings. Non seulement c'était la première fois à Francfort qu'une entreprise du secteur tertiaire - une assurance - pariait sur une altitude architecturale inhabituelle et ainsi abandonnait la solide tenue au sol qui avait été l'objet d'un consensus pendant très longtemps ${ }^{28}$, mais encore la forme changeait. En effet les architectes ont construit une «boite» de bureaux, dont les parties hautes classiques étaient supprimées. Même les arcades à l'étage du socle sortaient à peine comme des éléments marquants de l'image d'ensemble de la surface. Une peau extrêmement fine, légère et économisante, la façade dite "sandwich" était caractéristique d'une construction en squelette de béton armé29. À côté de la tour, les architectes installaient un bâtiment vertical dans le même style. Il rendait hommage à l'esthétique du pavillon, très populaire dans l'Allemagne d'après-guerre en raison de son association avec la mobilité. Ici se trouvait exclusivement le siège de la direction du groupe, tandis que la tour était louée à des locataires éminents tels que le consulat général de la Suisse.

Non seulement l'apparence très « américaine » de la tour mais surtout celle de la façade curtain wall, était sensationnelle - on la percevait comme extrêmement légère voire «planante ». L'emplacement de l'ensemble était encore plus provocant. Il bornait le coté ouest de la place de l'Opéra (Opernplatz) sur laquelle l'opéra lui-même, encore en ruines, attendait sa future "réanimation $»^{30}$. Mais avant tout, c'était la première fois qu'un building ${ }^{31}$ envahissait le quartier d'habitation du Westend et contrariait son caractère établi. La hauteur d'environ $70 \mathrm{~m}$ était au fond modeste car elle ne dépassait pas la tour de la cathédrale haute de $95 \mathrm{~m}^{32}$. Cependant elle était suffisamment importante pour 
qu'on perçoive comme une rupture dans la tradition, imposition d'un "solitaire " relativement haut dans un tel quartier, urbanisé à partir du milieu du XIX ${ }^{e}$ siècle. En effet il était encore inhabituel que la pression pour créer des surfaces de bureau s'empare systématiquement d'un quartier d'habitation proche du centre-ville ${ }^{33}$.

Les premières appréhensions et protestations - modérées comparées aux réactions futures- s'exprimèrent. Effectivement, la construction du Zürich-Haus fut de prime abord conçue comme un appel à l'industrie du bâtiment et au secteur tertiaire destiné à «mettre en valeur » le Westend. De plus, le terrain sur lequel on construisait le ZürichHaus avait été, fait à ne pas négliger, touché par les crimes de l'histoire allemande contemporaine, et donc chargé émotionnellement. Un palais de la célèbre famille Rothschild s'était trouvé ici ${ }^{34}$ : cet ensemble "aryanisé » en 1938 avait été gravement touché par les bombes durant la seconde guerre mondiale, et les ruines de la conciergerie furent démolies pour la construction du Zürich-Haus ${ }^{35}$. L'intrusion d'un building connoté comme international dans un quartier historique, occupant un lieu de mémoire chargé d'un souvenir précaire fut donc perçue encore plus comme une première violation de la tradition et comme l'expression d'un conflit d'intérêts. Les réactions émotionnelles vives n'étonnent guère. Surtout la façon de procéder, à savoir une libération du plan d'aménagement urbain, était perçue comme extrêmement inquiétante ${ }^{36}$.

Pour faire le bilan, il faut rappeler que, malgré les résistances civiles annoncées, le ZürichHaus, dont l'inauguration en 1962 fut perçue comme une véritable rupture, les réactions publiques et médiatiques se calmèrent un temps ${ }^{37}$. Plusieurs commentaires empathiques des grands journaux de Francfort tracèrent une image nettement positive de cette icône empreinte d'une modernisation économique, architecturale et urbaine. Cependant, la perception initiale du projet consistait en un préjudice pour la radicalisation ultérieure du développement, et annonçait le durcissement du front entre la ville (conjointement avec les investisseurs) et les habitants du quartier ${ }^{38}$. Peu après, dans le plan dit « des doigts » (Fingerplan) datant de 1968, le Zürich-Haus devait être rendu fonctionnel comme cadre pour la "viabilité économique» du Westend principalement le long de la Bockenheimer Landstraße. Donc il devait servir de base pour un ensemble qui émergeait déjà depuis quelques années ${ }^{39}$. La résistance de la population du quartier, celle des étudiants vivant à Bockenheim et des squatteurs augmentait considérablement. Elle culminait avec le combat des maisons (Häuserkampf) dans le Westend mené avec des moyens très durs qui trouvèrent leur apogée dans les années $1970-74^{40}$. La résistance portait sur l'indéniable restructuration socio-économique du quartier et sur la destruction croissante de sa substance traditionnelle ${ }^{41}$. Une charge émotionnelle de la scène urbaine du Westend en fut la conséquence. Mais curieusement le Zürich-Haus en tant que tel restait relativement exempt de ces luttes et ces drames. Il devenait à moyen-terme même une sorte de point d'orgue de la vie urbaine à proximité du centre-ville animé et proche du jardin public voisin, le Rothschildpark. En raison de son emplacement le building avec son horloge qui rythmait la vie urbaine fut exalté ${ }^{42}$ - peut-être plutôt $a$ posteriori, cela reste difficile à cerner - car il symbolisait un point d'intersection de différentes couches sociales. Même si on ne percevait pas le building comme «beau », on lui attribuait le plus souvent des critères positifs.

16 Voilà l'horizon contemporain devant lequel s'articulait le débat sur le destin et la signification du Zürich-Haus, et ce débat dura quasiment dix ans. La patrimonialisation du bâtiment restait, par contre, un véritable intermezzo, au moins au plan administratif. En effet, après que le propriétaire eut intenté une action officielle, contestant la protection 
accordée, le ministère de la culture du Land de Hesse abrogea le classement en tant que monument historique que le service des monuments historiques du Land avait attribué. Des réactions hautement émotionnelles du public s'exprimèrent alors fortement quand le propriétaire déclara sa volonté de détruire le building en $1995^{43}$. La ville officialisa la décision en 1998 après que le propriétaire eut menacé de quitter Francfort définitivement. La raison alléguée pour la destruction reposait sur des réclamations concernant le contrôle de la sécurité des bâtiments qui aurait nécessité des rénovations massives additionnelles du bâtiment.

Il est essentiel d'observer que la situation historique initiale s'inverse désormais. À l'époque l'intention d'empêcher la construction des buildings était encore dominée par les symboles et les outils d'une spéculation foncière mal aimée à l'intérieur du Westend. D'anciens opposants aux buildings, comme l'initiative action $d u$ Westend (AGW, Aktionsgemeinschaft Westend, fondée en 1969) qui avait été très active dans le quartier à la fin des années 60 et les années 70 deviennent alors des avocats du statu quo, donc de la préservation du Zürich-Haus. A l'époque du combat pour les maisons, cette représentation des intérêts des habitants du quartier agissait avec succès en matière de protection du patrimoine et de conservation du statut sociodémographique. Elle fut progressivement reconnue et devint un facteur considérable dans les débats sur l'avenir du Westend. Avec le temps le building Zürich-Haus a revêtu - pas uniquement pour les membres de cette initiative - une connotation émotionnelle positive, par exemple en tant que symbole de l'ère du miracle économique allemand et du re-essor rapide d'un nouveau Francfort l'ancien reposant en ruines juste à côté. Le building, de mieux en mieux accepté, fut finalement incorporé dans le canon des architectures dignes de protection. Il est vrai que cette perspective correspond à une perception de plus en plus positive que les habitants de Francfort ont adoptée globalement vis-à-vis des buildings et de la Skyline en tant que facteurs identificateurs ${ }^{44}$. Néanmoins il s'agit d'un cas bien particulier puisque l'ancien building fut défendu alors même par les sceptiques de l'architecture des tours modernes. De nombreuses lettres de lecteurs dans les quotidiens témoignent de ce refus qu'un ensemble originalement protégé fût livré à la destruction ${ }^{45}$. Des métaphores évoquant un enracinement organique du bâtiment dans le contexte urbain, et vice versa, d'une littérale " perte de la face urbaine $»^{46}$ dominaient dans ces contributions engagées dans le débat, donc des images drastiques de lésion, voire de meurtre symbolique. Mais en vain: le Zürich-Haus fut démoli effectivement dès août 2001. (ill.3) 


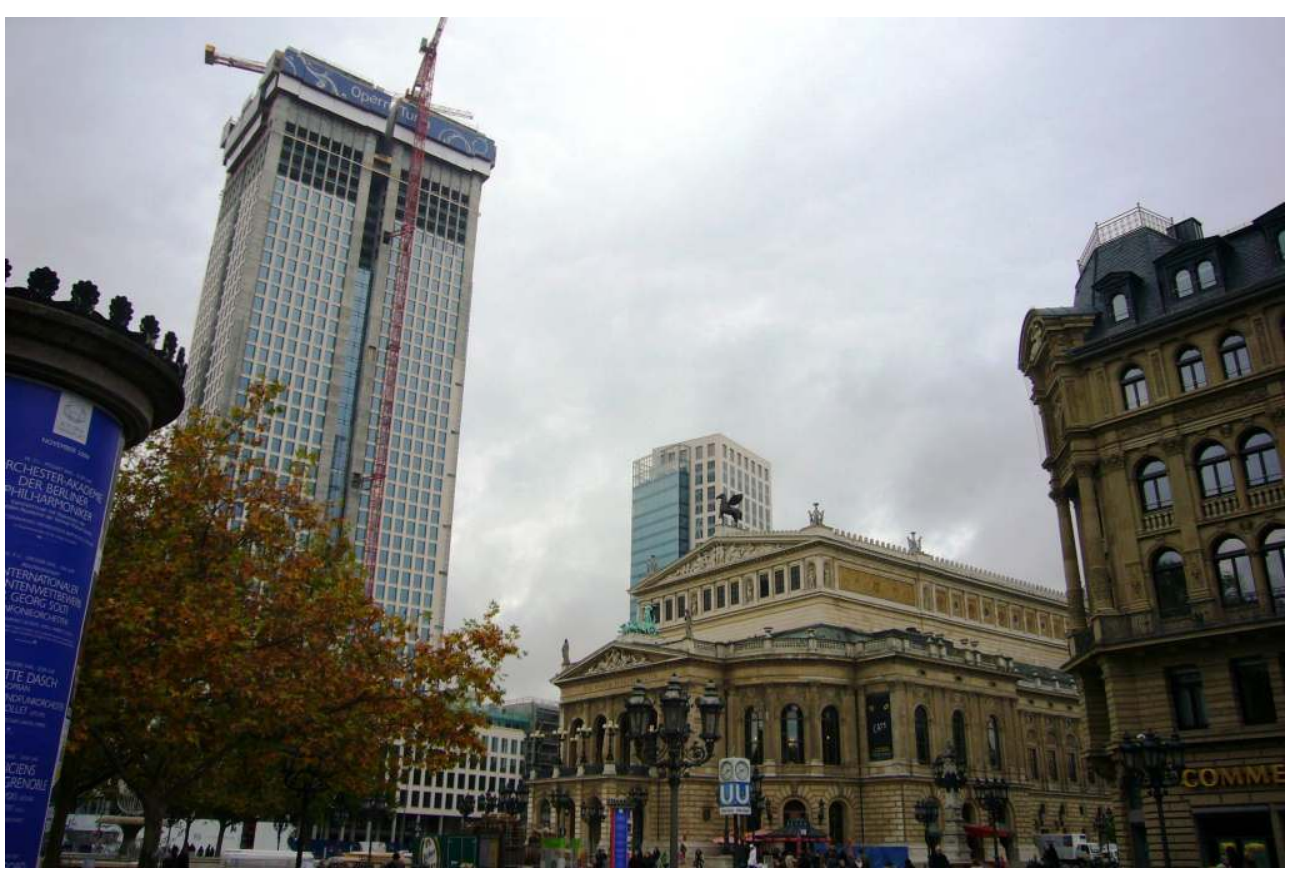

Cl. M. Dauss

La construction d'un nouveau bâtiment sur le terrain prestigieux, placé très avantageusement en marge de la cité, n'était pas moins discutée que la destruction de l'ensemble original qui fut et restait dans les esprits patrimonialisé. Les processus se prolongent jusqu'à nos jours alors qu'un nouveau building de bureaux, haut de $168 \mathrm{~m}$, a depuis peu été élevé à l'emplacement de l'ancien bâtiment : la dite Opernturm créée par l'architecte francfortois Christoph Mäckler. Son but n'est pas uniquement d'élever un building, mais aussi de clôturer cette place par une architecture en bord de parcelle, donc d'effectuer une mesure urbanistique ${ }^{47}$. L'importance de la durée d'élaboration du projet (plus de dix ans), n'est pas uniquement due à des problèmes techniques ou constructifs ; il faut ajouter à ces raisons l'intensité émotionnelle du débat mené par les politiques et les médias. Deux élections communales eurent lieu durant ce laps de temps. Elles fournirent le cadre à des scènes dramatiques : des changements de positions et des «trahisons » (du point de vue des habitants) du côté de la politique communale ${ }^{48}$. Des lettres de lecteurs fustigeaient les échecs des partis politiques. En effet, le nouveau projet devenait une véritable sorte de preuve de patience pour les décideurs communaux et pour le public. Même les partisans initiaux d'une destruction de l'ancien bâtiment - qui n'était pas unanimement apprécié par ses usagers ${ }^{49}$ - pensaient de plus en plus qu'on avait misé sur le « mauvais cheval ». La forte interdépendance de la construction des buildings et de la conjoncture économique a été responsable des interruptions et même du gel temporaire du processus. Le terrain resta inutilisé pendant cinq ans. L'entreprise qui avait jumelé la destruction et l'engagement de rester sur place, au centre-ville, se désista finalement en raison de problèmes économiques. Le trust restait tout simplement dans sa demeure «provisoire» à l'ouest, éloigné du centre-ville ${ }^{50}$. Un véritable roman policier mêlé d'événements singuliers dans l'histoire francfortoise se déroula ensuite. La ville devait prendre des mesures draconiennes contre la société d'assurance comme, par exemple, une garantie juridique de construire définitivement ${ }^{51}$. Après un long bras-de-fer, le trust 
présenta fin 2003 un investisseur new-yorkais qui avait déjà construit la tour de la foire ( Messeturm), une sorte d'emblème pour Francfort. C'est seulement après le transfert du projet à ce développeur américain que, malgré quelques turbulences ultérieures, la confiance pouvait être rétabliie ${ }^{52}$. (ill.4)

\section{4 : Opernturm, Francfort, à partir de 2007, Christoph Mäckler}

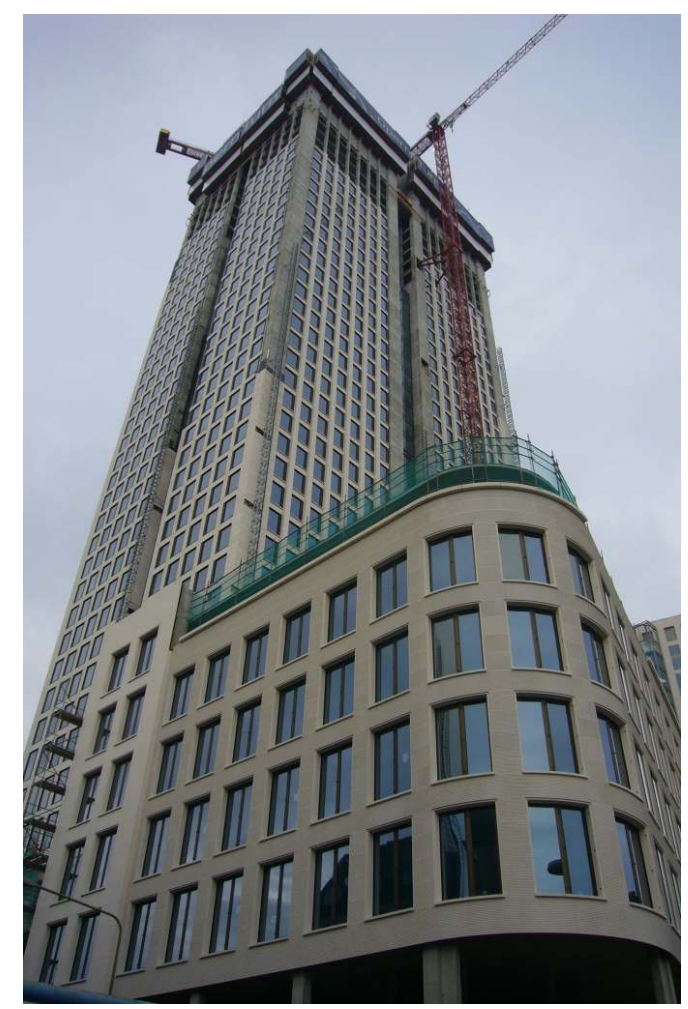

Cl. M. Dauss

Dans la perception du public, les hésitations et les progrès du projet devenaient donc une sorte de baromètre psychologique, avec ses hauts et ses bas, de l'industrie de la construction et de la conjoncture générale sur le site économique de Francfort ${ }^{53}$. Le projet faisait donc explicitement fonction, non seulement de référence mais aussi de stimulant pour le développement de la métropole économique que représente la ville de Francfort. En conséquence, les attentes et les déceptions temporaires des partisans du projet étaient très grandes. Or pour les critiques les analyses démasquant les difficultés étaient faciles ${ }^{54}$. De ce fait le projet de l'Opernturm est intégré dans un débat actuel hautement émotionnel sur les buildings et sur la physionomie de la ville de Francfort. Il porte sur le rapport entre les buildings « innovateurs > et les espaces traditionnels comme le Römer (une sorte de place de l'hôtel de ville) ${ }^{55}$, en particulier sur la concentration d'édifices du secteur tertiaire au centre-ville. Chaque nouvelle édition du plan général des buildings, sorti pour la première fois en $1953^{56}$ et juridiquement non-obligatoire, embrase le débat à nouveau ${ }^{57}$. La discussion sur les buildings ne peut pas contourner la question la plus fondamentale, celle de la hauteur-même des bâtiments - et l'importance de cette question augmente de génération en génération architecturale. De cette façon la hauteur de l'Opernturm était beaucoup plus cruciale encore que la dimension du Zürich-Haus ${ }^{58}$. On décida en 2001, après un intense lobbying de l'architecte ${ }^{59}$, une surélévation du bâtiment le portant des 90 mètres initiaux à approximativement 170 mètres. L'enjeu émotionnel du débat était 
proportionnel à la hauteur visée ; la fonction envisagée du building - servir pour la ville de nouvel emblème visible de loin - possédait donc un revers. Vu de plus près, ce point d'exclamation architectural possédait un fort lien avec la protection du patrimoine (un rapport négatif) et avec la continuité du paysage urbain (rapport à discuter). En effet la hauteur et la forme du bâtiment furent analysées intensément au niveau de son intégration à l'ensemble "traditionnel » de l'opernplatz (lui-même d'ailleurs en grande partie en reconstruction), et cela sous le rapport de l'ombre, de l'équilibre visuel, de l'accessibilité et de l'utilisation de l'espace public ${ }^{60}$. Le plan général des buildings de 1998 ( Hochhausrahmenplan) ne prévoyait pas une telle construction. Donc les ennemis du projet craignaient que le building, beaucoup plus haut qu'attendu, soit préjudiciable aux autres sites qui n'étaient pour autant pas mis en question dans le plan ${ }^{61}$. L'architecte faisait donc beaucoup d'efforts, soutenu par le journal de la $F A Z$, pour présenter le projet comme noyau de son concept de "guérison de la cité ». Le complexe devait éblouir par une façade en pierres apparentes ${ }^{62}$. Avant tout, les arcades commerciales latérales s'ouvrant sur l'Opernplatz devaient contribuer à créer le concept classique d'une place traditionnelle ${ }^{63}$. Aussi la nouvelle minceur de la tour - réalisée grâce à sa plus grande hauteur, en faveur de plus d'espaces verts - devait soutenir cette adaptation envisagée par l'architecte. (ill.5)

III. 5 : Opernturm, présentation sur le chantier

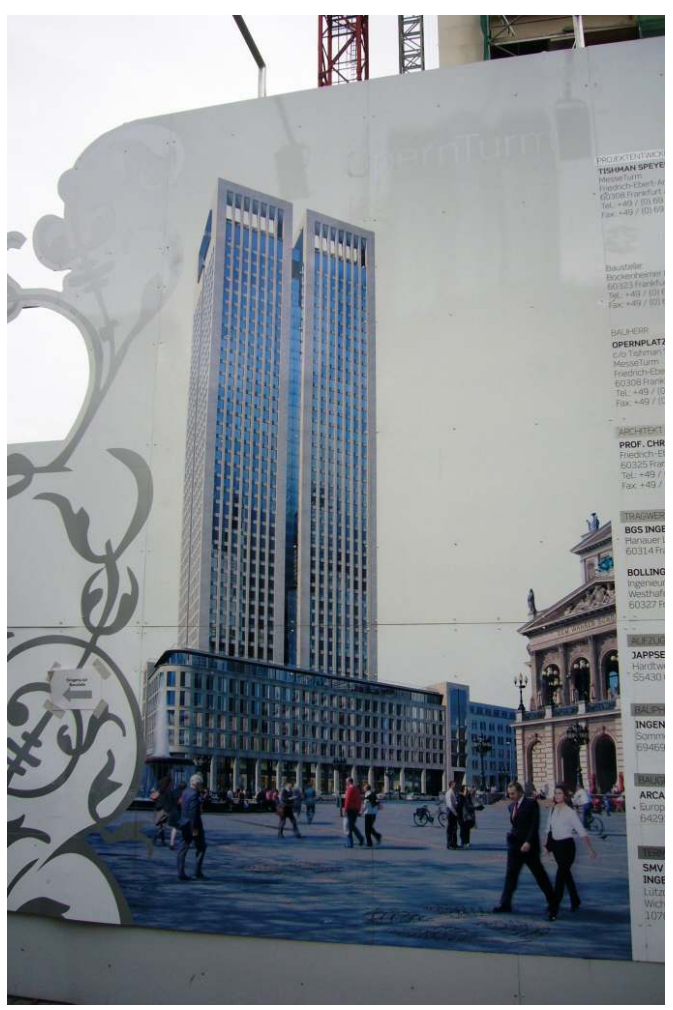

Cl. M. Dauss

Le concept opposé contre lequel l'architecte de l'opernturm argumente de manière hautement émotionnelle repose essentiellement sur la notion de premier modernisme fonctionnaliste et formaliste et sa continuité dans l'urbanisme d'après-guerre ${ }^{64}$. Des bâtiments comme le Zürich-Haus répondent selon lui à tous ces critères (négatifs) ${ }^{65}$. Ce maître d'œuvre (Mäckler sur Mäckler) ${ }^{66}$ le regarde comme témoin et acteur d'une utopie 
négative, en tant que non-lieu froid et marqué par une sensibilité manquante de matériaux $^{67}$. De même, pour d'autres lieux dans la ville, l'architecte propose une visualisation renforcée de couches historiques ensevelies sous des constructions ultérieures ${ }^{68}$. Des émotions - une identification positive à l'espace urbain, à ses lieux, ses ensembles centraux, et sa longue tradition - sont explicitement incluses dans un tel concept alternatif. Pour lui, une haute culture des matériaux assurant la dignité du vieillissement des bâtiments est porteuse de cette dimension émotionnelle; c'est pourquoi l'architecte a choisi une façade en pierres apparentes pour sa tour. Cette justification habile de Mäckler peut être déchiffrée dans une perspective dissidente comme une stratégie pour lier son projet au débat sur la physionomie de la cité déjà en cours (dans laquelle les lieux et les images d'identification sont centraux) ${ }^{69}$. Par cela les émotions positives du public peuvent être transmises sur un projet grandement précaire sous le rapport de l'identité historique et visuelle. Le fait de légitimer la destruction et l'innovation comme une sorte de préservation de la tradition est une interprétation spécifique et stratégique de la thèse générale - qui semble plus valide - que l'innovation est la vraie tradition, le «sort» d'une ville comme Francfort ${ }^{70}$. L'ambition, donc une motivation profondément psycho-émotionnelle, était d'ajouter à sa biographie architecturale un building, encore manquant, excédant 100 mètres, au centre de Francfort et de s'allier aux intérêts des investisseurs, cette ambition est portée vers une mission historique supérieure ${ }^{71}$.

Les adversaires ${ }^{72}$ et critiques de l'architecte n'ont pas uniquement démasqué cette motivation sous-jacente ${ }^{73}$, mais ils ont aussi critiqué le traditionalisme nostalgique et les prémisses discutables de l'approche théorique de l'architecte, son but et les moyens prévus. En effet, c'est son intention principale d'assurer une harmonie ressentie dans la communauté urbaine par un artisanat traditionnel et par une «bonne » esthétique des matériaux, donc par un idéalisme objectivé ${ }^{74}$. De tels concepts ont été réfutés - pas à tort - en tant que concepts simplificateurs ${ }^{75}$. Une seule évidence émerge de ce débat: c'est surtout la négociation permanente de l'identité urbaine dans une ville comme Francfort marquée autant par une forte migration que par une grande fluctuation et donc par un séjour inconstant d'une grande partie de la population. La coexistence éclectique de l'architecture caractérise la ville nécessairement par une discontinuité d'échelle et de proportions, typique de nos villes qui resteront toujours des villes d'après-guerre. Assimilée à une harmonie et à une homogénéité, la fiction d'une identité considérée comme statique doit en revanche paradoxalement omettre l'inépuisable émotivité si son but doit se manifester dans la stabilité affective des citadins. Avant tout, cette proposition de l'architecte Mäckler d'introduire une charte prescrivant la physionomie des bâtiments érigés en ville pour assurer la continuité visuelle et la cohérence sociale par des normes formelles et matérielles peut être et a été repoussée avec un argument principal ${ }^{76}$ : plus que n'importe quelle charte formaliste, il aurait dû y avoir une protection du patrimoine effective et respectée, vrai catalyseur du consensus urbain et social. 


\section{NOTES}

1. Le terme de monument (Denkmal) vise d'abord un caractère mémoriel qui doit être garanti pour une longue durée. Celle-ci est normalement évoquée par la massivité, la grandeur ou la hauteur des constructions. Monumentalité dans l'espace et dans le temps y sont complémentaires. Si la sémantique mémorielle se perd, les constructions tendent à devenir des monuments d'eux-mêmes, de leur genèse ou de leur propre présence, donc elles deviennent autoreferentielles. Justement, la force des monuments modernes à s'imposer d'une telle façon peut être grande car souvent ils arrivent à se graver littéralement dans la mémoire visuelle et à assurer leur propre présence. En outré, ils prennent des fonctions de structuration ou de repérage dans les espaces urbains dans lesquelles ils orientent l'individu ou qu'ils représentent en tant qu'emblème. Cf. pour la fonction des monuments : Jean-Michel Leniaud, L'Utopie française. Essai sur le patrimoine, Paris, Mengès, 1992, 180 p. ; id., Les Archipels du passé. Le patrimoine et son histoire, Paris, Fayard, 2002, 360 p.

2. Rainer Stomme/Dieter Mayer-Gürr, Hochhaus. Der Beginn in Deutschland, Marburg, Jonas, 2002, $261 \mathrm{p}$.

3. Marianne Rodenstein, «Von der 〈Hochhausseuche > zur 〈Skyline als Markenzeichen > - die steile Karriere der Hochhäuser in Frankfurt am Main », dans : id. (éd.), Hochhäuser in Deutschland. Zukunft oder Ruin der Städte? Bonn, Kohlhammer, 2000, 281 p., ici : p. 15-70, ici : p. 67. L'auteur constate de façon prémonitoire qu'un jour, la protection du patrimoine pouvait devenir l'enjeu principal dans le débat francfortois sur les buildings.

4. Matthias Alexander, "Hochhausprojekte gesucht", FAZ Sonntagszeitung, 03.7.2005, $\mathrm{n}^{\circ} 26$, p. V13 ; id., « Das letzte Jahr mit Europa-Rekord », FAZ, 03.01.2007, n² 2, p. 38.

5. Manuel Cuadra, «Ursprünglich war die Skyline nur ein Nebenprodukt », FAZ Sonntagszeitung, 27.06.1999, n² 25, p. 16.

6. Matthias Alexander, « Die gereifte Hochhausstadt», $F A Z, 31.10 .2006, \mathrm{n}^{\circ} 253$, p. 51.

7. «Stadtreparatur wie im Bilderbuch », FR, 27.01.2007, p 13.

8. Ulf Jonak, Die Frankfurter Skyline. Eine Stadt gerät aus den Fugen und gewinnt an Gestalt, Frankfurt/ New York, Campus, 1997, 157 p.

9. Werner Durth/Nies Gutschow, Träume in Trümmern. Planungen zum Wiederaufbau zerstörter Städte im Westen Deutschlands 1940-1950, 2 tomes, Brunswick/Wiesbaden, Vieweg, 1988, 329 et 331 p.

10. Building de l'AEG (AEG-Hochhaus), conçu initialement comme ministère fédéral du travail, inauguré en 1961, détruit en 1999 ; building de la télécommunication (Fernmeldehochhaus), inauguré en 1954, détruit en 2005, et maison Zürich (Zürich-Haus). Cf. «Der stille Abschied von der Nachkriegszeit ", FR, 10.04.2007, p. 33.

11. Des architectes comme Christoph Mäckler - qu'on va introduire plus loin - ne concèdent généralement pas plus de 30 ans d'existence aux buildings.

12. Cela peut étonner car la critique dissidente et émancipatrice du fonctionnalisme architectonique et urbanistique fait partie essentielle du discours d'après 1968.

13. «Zürich-Haus: Mehrheit für den Abriss sicher », FAZ, 19.03.1998, nº66, p. 46.

14. Mon article repose sur une étude de la $F A Z$ et de la $F R$ entre 1994 et respectivement 1998 et aujourd'hui. Dans une version de ce texte modifiée par un focus différent («Innovative Höchstwerte? Hochhausbau und -abriss in Mainhattan », Christian Freigang/Markus Dauss/ Evelyn Brockhoff (ed.), Das ,neue' Frankfurt. Innovationen in der Frankfurter Kunst vom Mittelalter bis heute (Vorträge der 1. Frankfurter Bürger-Universität), Frankfurt, Kramer 2010, p. 124-143) j'ai 
inclue une étude de documents d'archives, de l'archive municipal et de la caisse des monuments historiques de Francfort (Denkmalamt).

15. Pour ces concepts cf. Joachim Fischer, «Die Bedeutung der Philosophischen Anthropologie für die Architektursoziologie", Karl Siegbert Rehberg (éd.), Soziale Ungleichheit - Kulturelle Unterschiede. Verhandlungen des 32. Kongresses der Deutschen Gesellschaft für Soziologie in München 2004 , Frankfurt/New York, Campus, 2006 (CD-ROM), p. 3417-3429.

16. Pour cette phase cf. Rodenstein, op. cit., p. 20s., 33s.

17. Ibid., p. 21s.

18. Rodenstein (op.cit.) a présenté une vue d'ensemble très solide et bien documentée sur l'architecture des buildings à Francfort. Elle se focalise avant tout la planification et la sociologie urbaine. Nous nous référons continuellement à cet article.

19. Le tableau architectural de l'époque nazie est bien plus complexe que cela, mais l'architecture de représentation est naturellement la plus visible.

20. Cela vaut en général, mais par rapport aux caractéristiques architecturales et urbaines plus concrètes des buildings, le magistrat, généralement accueillant pour l'architecture des buildings, était sceptique: les constructions dans un "style américain », donc très hautes et denses, n'étaient pas souhaitables. Cf. Rodenstein, op. cit., p. 22-31.

21. Roland Burgard, «Einziges und intaktes und zusammenhängendes Dokument des Wirtschaftswunders », FR, 24.07.2003, p. 28.

22. Pour la forme et signification des toitures des buildings francfortois : Dieter Bartetzko, «Die schönsten Krone Frankfurts ist eine imaginäre »; FAZ Sonntagszeitung, 13.10.1996, nº 41, p. W1 ; id., « Einfach nur flach oder die Krönung der Hochhäuser », FAZ Sonntagszeitung, 30.08.1998, n 35, p. W9.

23. Rodenstein, op. cit., p. 30.

24. Pour cela et ce qui suit : ibid., p. $25 f$.

25. Matthias Alexander, « Oben wird es eng », FAZ, 02.08.2001, n 177, p. 11.

26. Rodenstein, op. cit., p. 31.

27. Santifaller, "Steht das Zürich-Haus schief?», Dieter Bartetzko (éd.), Sprung in die Moderne. Frankfurt am Main. Die Stadt der 50er Jahre, Frankfurt am Main/New York, Campus 1994, 160 p., p. 80-99, ici : p. 90-96; Rodenstein, op. cit., p. 30s.

28. La première génération des buildings a été vouée aux soucis de rentabilité plus qu'aux aspects symboliques. Dans un temps où les affaires reposaient encore largement sur un contact direct au client, cela ne semble pas trop étonnant. Dans la mesure où les opérations de crédit dépendent plus de l'internationalisation et de l'anonymat la connotation des buildings en verre, souvent inaccessibles, semblait plus apte. Rodenstein, op. cit., p. 39s.

29. Elle est une combinaison de différents matériaux - qui cependant créait des problèmes thermiques immenses. Cf. Ernst Wegener, « Beginn der 〈 Entwicklung der City ins Westend 〉 - das Zürich-Haus am Operplatz », Detlef Janik (éd.), Hochhäuser in Frankfurt. Wettlauf zu den Wolken, Frankfurt am Main, Societäts-Verlag, 1995, 111 p, p. 36.

30. L'opéra ne fut inauguré qu'en 1981 après une reconstruction coûteuse.

31. À partir de 1955 toutes les constructions francfortoises dépassant 22 mètres ou ayant plus de 8 étages sont définies comme building.

32. Sur la rive sud du Main le Henninger Turm surplombe la tour de la cathédrale. Elle est plutôt un bâtiment industriel qu'un véritable building, haut de $120 \mathrm{~m}$.

33. Rodenstein, op. cit., p. 34.

34. Le terrain sur lequel la famille de Rothschild avait fait construire à partir de 1832 un jardin anglais fut acheté par la ville en 1938 pour un prix beaucoup plus bas que le prix courant. Après la deuxième guerre mondiale les négociations entre les anciens propriétaires et la ville reprirent. Le prix fut re-estimé, et les héritiers de la famille obtinrent la restitution d'un tiers du terrain. En 1960 le dernier acte transactionnel eu lieu. Après une autorisation de construire, les Rothschild 
vendirent l'ensemble au konzern Zürich-Versicherung et la Berliner Handelsgesellschaft (BHG) qui y ont fait construire leurs sièges. Cf. « Sanfte Kurven. Wenn der Opernturm fertig ist, darf auch der Rothschildpark wieder wachsen ", FR, 22.10.2008, p. 1 ; Rodenstein, op. cit., p. $35 f$.

35. Les vestiges du palais ont été détruits en faveur de la centrale de la BHG (1960-66) que le trust a commandé à l'architecte Sep Ruf, figure éminente de la scène architecturale allemande aprèsguerre.

36. Pour les détails de la procédure : Rodenstein, op. cit., p. 36f.

37. Pour cela et ce qui suit : Santifaller, op. cit., p. 80-99.

38. Detlef Janik, « Wie die Hochhäuser nach Frankfurt kamen », idem, op. cit., p. 9-12, ici : p. 10.

39. Rodenstein, op. cit., p. 38, 43-46. Le Fingerplan avait été conçu comme moyen de planification urbanistique et de canalisation des intérêts divergents, mais par les habitants et les critiques il fut perçu comme largement insuffisant et intéressé. Des «arrangements» entre ville et investisseurs restèrent à l'ordre du jour jusqu'aux années 80 . Une des conséquences ne fut pas uniquement la pérennisation des initiatives de protestation, mais à long terme aussi l'enracinement du parti des Verts, représenté au parlement municipal pour la première fois en 1981.

40. Ces événements ont rattrapé il y a quelques années le ministre des Affaires étrangères de l'époque, le vert Joschka Fischer.

41. Rodenstein, op. cit., p. 37s.. Les essais de la ville pour empêcher la spéculation foncière sur le quartier n'aboutirent pas.

42. Matthias Alexander, " 170 Meter hoch, schlank, gelb, ohne Uhr ", FAZ, 06.05.2002, n 104, p. 55 .

43. Ibid.

44. Id. « Auf den zweiten Blick. Das Verhältnis der Frankfurter zu ihren Hochhäusern besserte sich erst in den achtziger Jahren ", FAZ, 06.05.2001, $\mathrm{n}^{\circ} 18$, p. B1. Les multiples mesures de compensation de l'ère du maire Walter Wallmann (1977-86), focalisant l'attention sur le Römer et sur la rive des musées, ont sûrement contribué largement à la revalorisation des buildings. Mais en outre les changements de leur physionomie à partir de l'époque postmoderne peuvent être un facteur qui a influencé l'évolution de l'image publique des géants architectoniques.

45. Naturellement ont craignait aussi une dégradation ultérieure de la qualité de vie dans le Westend. Yvette Mazzante, «Anfang vom Ende », FAZ, 22.11.2001, p. 60.

46. Stephan Kyrieleis, « Abriss », FR, 09.05.2003, p. 26.

47. « Grundstein für Opernturm gelegt », FAZ, 05.09.2007, n²06, p. 44.

48. Quelques exemples pour une telle critique : Renate A. Wilson, «Nicht gefallen lassen », $F A Z$, 15.11.2001, p. 62 ; Daniel Güthert , « Taschenspielertrick », FAZ, 17.11.2001, p. 76.

49. Déjà la première génération des buildings à Francfort n'avait pas toujours su convaincre leurs utilisateurs. Rodenstein 2000, p. 32.

50. Matthias Alexander, « Thisman Speyer baut Zürich-Turm », FAZ, 15.11.2003, n 266, p. 61.

51. Claudia Michels, "Schmales Hochas », FR, 15.11.2003, p. 29.

52. "Zürich-Areal : Investoren verpflichten sich zur Bebauung ", $F A Z, 06.07 .2004, \mathrm{n}^{\circ} 154$, p. 39. Lors du transfert du projet à l'investisseurs New-yorkais Tishman Speyer la catégorie de la confiance mutuelle était omniprésent. Jerry Speyer est issu d'une famille des émigrés francfortois qui ont fui la terreur Nazie. («Lückenschluss an einem der schönsten Plätze der Stadt », FAZ, 23.01.2007, $n^{\circ} 19$, p. 44). Malgré cette avance de confiance la ville et l'investisseur ont conclu un contrat obligeant le premier à construire dans un délai d'une demi-année après l'obtention du permis de construire. Entre-temps Tishman Speyer ne croyait plus que le building soit réalisable et voulait se borner à une construction seulement le long la marge de la parcelle, mais désormais le building est en construction. Cf. Claudia Michels, "Opernplatz: Der Turm wächst. ", FR, 01.02.2005, p. 34 ; Claus-Jürgen Göpfert, «Frankfurt beendet Stillstand bei Zürich-Areal », FR, 07.07.2004, p. 20 ; « Arkadenbau soll Brache am Opernplatz füllen », FR, 18. April 2006, p. 14. 
53. «Zürich-Areal wird vorübergehend zum Parkplatz », $F A Z, 06.12 .2002$, n 284, p. 55 ; Rainer Schulze, «Einige hundert Meter Zuwachs für die Skyline», FAZ, 08.01.2008, n 6. p. 41 ; ClausJürgen Göpfert, « Notfalls ein Hotel statt der Büros », FR, 19.02.2004.

54. Claudia Michels, «Stadt zeigt der Zürich-Versicherung die Muskeln », FR, 08.07.2003, p. 24.

55. On peut comprendre l'Opernturm comme parallèle ou même antipode à la banque centrale de l'Europe conçue par COOP Himmelb(l)au qui suit un langage architectural très différent. Le marché couvert de Martin Elsaesser - monument historique de l'ère moderne - y rencontre une tour déconstructiviste, une configuration engendrant beaucoup de conflits. Christian Thomas, «Bauen auf Kompromisse. Zwei Beispiele aus Frankfurt », FR, 07.07.2006.

56. Le premier plan lancé par Herbert Boehm, chef de la planification communale, en 1953 était appelé Hochhausplan, pas encore Hochhausrahmenplan.

57. Concernant le rôle du plan: Matthias Alexander, « «Schon an die alten Vorgaben hat sich niemand gehalten» ", FAZ, 24.08.2006, n 196, p. 41 ; « Empfindliche Kürzungen für Campanile und Allianz-Turm », FAZ, 19.04.2008, n 92. p. 54 ; Matthias Arning/Jürgen Schultheis, « Das wird was. Karte entfaltet die Stadtentwicklung Frankfurts ", FR, 24.10.2008, p. 3.

58. Reinhild Spiess, «Am Nasenring », FAZ, 21.11.2001, p. 62.

59. La SPD a voté contre, la CDU et les Verts pour la surélévation. Les derniers n'avaient pas opté pour la destruction du Zürich-Haus, mais ils voyaient dans la surélévation du bâtiment une chance pour gagner plus d'espace vert. "Mehrheit für 168 Meter hohen Turm am Opernplatz », FAZ, 21.11.2001, p. 59.

60. L'architecte a su intégrer ces critiques dans sa propagation d'une surélévation du bâtiment qui gagnait en minceur. Günter Mick, «Wahrzeichen einer neuen Identität », FAZ, 14.05.2007, n -111, p. 43.

61. Magda Thelen, « Nicht zu tolerieren », FAZ, 06.12.2001, p. 66.

62. Non sans raison l'esthétique de la transparence á été déchiffrée de plus en plus comme purement rhétorique. Alexander, « Glas-Wahn », FAZ, 01.04.2004, p. 41.

63. «Städtisches Leben auch an der Westseite des Opernplatzes », FAZ, 05.05.2001, n 104, p. 75.

64. Erst Lackner, «Christoph Mäckler. Lieber eine Bauhütte als eine Architektur-Fabrik », FAZ Magazin, 04.07.1997, n 905, p. 6.

65. Klaus-Jürgen Göpfert, « Der Störenfried », FR : 08.03.2003, p. 29.

66. Läckner, op. cit.

67. Pour cela et ce qui suit: « 〈Politiker haben oft keine Ahnung von Architektur». Christoph Mäckler wünscht sich mehr Geschichtsbewusstsein in der Baukultur », (Matthias Alexander und Günter Mick im Gespräch mit dem Architekten), FAZ, 03.04.2006, n 79, p. 47.

68. «Im Gespräch : Der Architekt Christoph Mäckler. «Stadtgestaltung kann nicht nach Moden gehen» », FAZ, 15.10.2008, n 241, p. 45.

69. Un bref survol : Matthias Alexander, « Baubeginn frühestens Anfang 2010 », FAZ, 11.12.2006, n - 288, p. 43 ; Rainer Schulze, «Der schmale Grat zwischen Alt und Neu », FAZ, 23.05.2008, n 118, p. 48 ; Birgit Ochs, «Die neue Sehnsucht nach dem Alten », FAZ Sonntagszeitung, 25.05.2008, $\mathrm{n}^{\circ} 21$, p. V17.

70. Pour cela cf : les conférences publiques de la série : Das 'neue' Frankfurt vom Mittelalter bis heute - Innovationen in der Frankfurter Kunst, initiée par l'institut de l'histoire de l'art de l'université de Francfort et l'institut pour l'histoire municipale, octobre 2008 - janvier 2009.

71. Dans la City West, en marge de la ville, Mäckler avait déjà construit entre 1996 et 2001, le building Scala, haut de 18 étages

72. L'AG Westend était à la tête du rejet d'une tour de $168 \mathrm{~m}$. Rudolf Dederer, lettre de lecteur dans la $F R, 27.01 .2007$, p. 30. 
73. Il semble délicat que Mäckler critique des allures des stars de l'architecture et leur refus de se plier à des idées d'ensemble et qu'il se puisse se croire exempt d'une attitude semblable. Idem, «Zerstörungen im Namen der Avantgarde », FAZ, 22.11.2006, n 272, p. 41.

74. «Mäckler: Gestaltungssatzung für prominente Plätze der Stadt», FAZ, 13.11.2000, n 264, p. 63. Christoph Mäckler, « Der Herrenschuh », FAZ, 13.03.2001, n 61, p. 64.

75. Manuel Cuadra, «Baukultur bitte ohne Korsett », FAZ, 04.05.2001, $\mathrm{n}^{\circ} 103$, p. 69. Cf. aussi les contributions de Jürgen Engel, Vittorio M. Lampugnani und Michael Kummer sous la rubrique : "Ein Stadtbild, dem historische Anknüpfungspunkte fehlen»; außerdem: Dieter von Lüpke, «Das Urteil über architektonische Qualität ist zeitgebunden», $F A Z, 13.06 .2001, \mathrm{n}^{\circ} 135$, p. 68 ; Florian Pollmann, « Äußerst desolate Plätze », FAZ, 03.07.2001, n 151, p. 54 ; Karl Richter, « Zum Gedächtnis eines Ortes gehört auch die radikale Stadterneuerung », FAZ, 04.04.2001, p. 65.

76. "Auf der Skyline gründet der Stolz. Fritz Novotny zur Architektur-Debatte über Gestaltungssatzungen. ", FAZ, 27.11.2000, n² 276, p. 65.

\section{RÉSUMÉS}

Le rapport entre patrimoine et émotion peut être illustré en se fondant sur l'architecture des buildings. En effet un lien évident peut être établi entre émotion et hauteur des constructions. En tant que représentation emblématique de la construction moderne, la notion de hauteur se trouve plus aisément chargée de points culminants émotionnels plutôt que de valeurs sublimées de l'ancienneté telles que les monuments les recèlent. Or, depuis peu, l'architecture des buildings et les discours concernant la protection des monuments semblent se rapprocher de plus en plus. En Allemagne la dimension émotionnelle des buildings peut être suivie de façon paradigmatique et plus lisible qu'aux États Unis dont l'architecture en hauteur est souvent dite " naturelle ». Déjà la réception presque euphorique de l'architecture d'outre-Atlantique des buildings dans les années 20 en Allemagne - à cette époque encore largement conçue comme un devoir public provoquait un scepticisme important. Par conséquent le débat restait vivant et clairement explicité. Et même si après la deuxième guerre mondiale l'influence américaine germait bien sur les terres allemandes - surtout dans certaines villes - les buildings ont toujours été l'épicentre de luttes fortement émotionnelles pour le monopole d'interprétation que détient le discours architectural et urbain, et cela reste toujours d'actualité. Les buildings considérés en tant que véritables monuments, concept qui engloberait alors leur protection, demeurent très rares. À Francfort on comprend à quel point tant la construction que la disparition des buildings ont suscité d'émotions. Cela vaut d'autant plus que les buildings y acquièrent actuellement un statut objectif, c'est-à-dire institutionnalisé, de monument historique. Le cas que l'article présente est exemplaire pour exprimer la convergence des discours sur le patrimoine et sur la dimension émotionnelle. Il est essentiel que l'architecture de la reconstruction du Francfort d'après-guerre - qui a duré jusqu'aux années 70 - puisse maintenant faire valoir une valeur d'ancienneté, ce que les nouveaux projets de construction reflètent d'ores et déjà. Les démolitions sont à l'ordre du jour, surtout celles de la première génération des buildings qui s'est terminée vers la fin des années 50. La révolution des tours « mange » alors ses enfants au moment même où ils sont assez vieux pour être considérés comme monuments. Cette nouvelle situation s'applique surtout à la démolition du building de la mutualité Zürich (Zürich-Haus; construit en 1958) en 2001, remplacée par la tour de l'Opéra (Opernturm) en construction depuis 2007, terminé en 2009. Les débats autour de ces changements au centre-ville de Francfort sont fortement émotionalisés pas 
uniquement en raison de la hauteur des buildings, mais aussi parce que les notions de monument classique et de patrimoine sont impliquées.

The relationship between heritage and emotion can be illustrated by looking at the architecture of modern buildings. A link can indeed be established between emotion and the height of a building. As an emblematic representation of modern architecture, the notion of height can be more easily charged with culminating emotional points than with sublimated values of 'oldness', such as those that are present in historic monuments. Over the last few years, the architecture of modern buildings and the discourse concerning the protection of monuments seem to be coming closer and closer together.

In Germany the emotional dimension of buildings can be followed in a paradigmatic way and is more easy to interpret than in the United States where tall buildings are often said to be 'natural'. Already, during the 1920s, the almost euphoric reception of American-style buildings in Germany-at this time still largely conceived as a public duty-provoked considerable scepticism. Consequently, the debate remains lively and clearly formulated. And even if after the Second World War the American influence took root in German soil, particularly in certain cities, tall buildings were at the centre of highly emotional debates around the monopoly of interpretation held by architectural and urban discourse, and that is still true today. Modern buildings considered as authentic monuments, a concept which implies their protection, remain very rare.

Der Zusammenhang von patrimoine und Emotion kann anhand der Hochhausarchitektur exemplarisch veranschaulicht werden. Denn als markanteste Verkörperung eines dezidiert ,modernen' Bauens liegt die Aufladung der Türme mit emotionalen ,Spitzenwerten' näher als die Akzentuierung eines würdigen ,Alterswertes', den der Denkmalbegriff meint. Aber auch zwischen Hochhausarchitektur und Denk-mal(schutz)diskurs besteht ein enger und neuerdings intensivierter Zusammenhang.

Die emotionale Dimension von Hochhäusern lässt sich paradigmatisch, und zwar viel deutlicher als in den USA mit ihrer vermeintlich ,naturwüchsigen' Hochhausarchitektur, in Deutschland verfolgen. Schon die frühe, teilweise euphorische Rezeption der überseeischen Hochhausarchitektur in den 20er Jahren des 20. Jahrhunderts - damals noch primär eine öffentlich konzipierte Bauaufgabe - hatte in Deutschland auch große Skepsis hervorgerufen. Entsprechend lebhaft verlief auch in der Folge die Debatte. Zwar fiel dann nach dem Zweiten Weltkrieg der transatlantische Keim generell auf einen viel fruchtbareren Boden - in einigen Städten schien er sogar ganz besonders aufnahmefähig zu sein; aber es galt und gilt weiterhin, dass Hochhäuser Nukleen hochgradig emotionalisierter Kämpfe um Deutungshoheiten innerhalb des architektonischen und urbanistischen Diskurses sind. Effektiv geschützte Denkmäler sind dabei die wenigsten Hochhäuser.

Besonders deutlich lässt sich das bisher Gesagte in Frankfurt nachvollziehen. Die Entstehung, aber auch das Verschwinden von Hochhaustürmen schlagen hier hohe emotionale Wellen - und dies umso mehr, als der ,objektive', d.h. institutionalisierte, Status des (geschützten) Denkmals, der immer Aushandlungsprozessen unterliegt, sich mit Bezug auf Hochhäuser noch in einer besonderen Etablierungsphase befindet. Entscheidend ist, dass die Architektur des Wiederaufbaus im Frankfurt der Nachkriegszeit, die man bis in die 70er Jahre hinein datieren kann, heute bereits einen Alterswert zu reklamieren vermag, der neuerdings mit Neubauprojekten kollidiert. Abrisse sind nämlich an der Tagesordnung, vor allem solche der sog. ersten Hochhausgeneration, die mit dem Ende des 50er Jahre ausklingt. Die Hochhausrevolution frisst also nun ihre eigenen Kinder, und das just in dem Moment, in dem diese erstmals potentiell denkmalwürdig werden.

Für den im Artikel auf seine emotionale Dimensionierung hin untersuchten Abriss (2001) des Hochhauses der Zürich-Versicherung, das durch den sog. Opernturm (seit $2007 \mathrm{im} \mathrm{Bau)} \mathrm{ersetzt}$ wird, gilt diese neuartige Konstellation ganz besonders. Nicht nur wegen der Bauhöhe, sondern 
auch, weil der Denkmal(schutz)begriff impliziert war, geriet die Debatte über diesen punktuellen Stadtumbau so emotional.

\section{AUTEUR}

\section{MARKUS DAUSS}

Markus Dauss est né en 1974. De 1995 à 2000, il suit des études d'histoire et de l'histoire de l'art à l'Université Carl von Ossietzky d'Oldenbourg et à l'Université de Paris IV - Sorbonne ; il soutient un magister artium en histoire de l'art (M.A.) et une maitrise d'histoire en histoire sous la direction de Jean-Pierre Chaline. Il poursuit son cursus de 2000 à 2004par une thèse de doctorat (thèse en cotutelle) à la TU de Dresde et à l'École Pratique des Hautes Études Paris sous la direction de JeanMichel Leniaud, Karl-Siegbert Rehberg et Gerd Schwerhoff. Boursier postdoctoral au collège des gradués „Klassizismus und Romantik“ à l'Université Justus Liebig de Giessen entre 2004 et 2006, il devient à cette date maitre de conférences (wissenschaftlicher Mitarbeiter) au département de l'histoire de l'art à l'Université Johann Wolfgang Goethe de Francfort. Ses publications principales sont : Identitätsarchitekturen. Öffentliche Bauten des Historismus in Paris und Berlin (18711918), Dresde, Thelem, 2007 ; avec Ralf Haekel (dir.), Leib/Seele - Geist/Buchstabe. Dualismen in der Ästhetik und den Künsten um 1800 und 1900, Würzburg, Königshausen und Neumann, 2009 ; Bibliotheken als gebaute Ordnungen des Wissens, dans : Kunst und Wissen. Beziehungen zwischen Ästhetik und Erkenntnistheorie im 18. und 19. Jahrhundert, sous la dir. d'Astrid Bauereisen, Stephan Pabst et Achim Vesper, Würzburg, Königshausen und Neumann, 2009, p. 257-282. 